\title{
Assessment and management of dysphagia and achalasia
}

\begin{abstract}
Dysphagia is a common symptom which can vary in severity and aetiology; at one end, it can be a benign inconvenience, on the other, there can be serious morbidity associated with malnutrition. It is crucial to identify those with mucosal and structural disease, including malignancy as a priority first. Reflux disease is commonly a culprit and treating empirically with acid reducing medicines should follow exclusion of organic disease. Other benign conditions (including eosinophilic oesophagitis) should be considered. The clinical assessment of dysphagia begins with a detailed history and a focus on symptom severity as well as the pre-test probability of a given condition. Tests are then directed at assessing function, and should employ both high-resolution manometry and barium studies. For motility disorders, begin by assessing the oesophago-gastric junction for obstruction (eg achalasia), followed by oesophageal body function. The latter is divided into major and minor motility disorders. Treatment is directed according to the dysmotility phenotype and is based upon background fitness, age and appetite to intervention. Invasive treatment for achalasia is aimed at disrupting the lower oesophageal sphincter muscle while that of oesophageal body disorders is directed at reducing hypercontraction, improving peristalsis or reducing symptoms.
\end{abstract}

\section{Introduction}

Dysphagia is the subjective awareness of impairment in the swallowing of saliva, liquid or solid food, and is a common indication for referral to specialised clinics. ${ }^{1}$ As it is considered an alarm symptom, assessment is required to exclude malignancy, mucosal and structural disease. ${ }^{1}$ Benign disorders can also be associated with significant morbidity and impaired quality of life. Broadly, it is divided into oesophageal and oropharyngeal dysphagia; the latter more prevalent in elderly patients, commonly related to neurovascular diseases. ${ }^{2}$ Oropharyngeal dysphagia is characterised by a difficulty in initiating swallows and aspiration, and is beyond the focus of this article. The aim of our review is to provide a practical clinical approach to the evaluation and management of non-malignant oesophageal dysphagia.


EMMS, Nazareth, Israel and Bar Ilan University, Safed, Israel; Bgastroenterology consultant, University College Hospital, London, UK

\section{Symptoms of dysphagia}

Tolerability of swallowed food/fluid is a poor predictor of underlying disease; however, dysphagia accompanied with periods of resolution is more likely to be associated with motility dysfunction, while a mechanical disorder (eg obstruction due to a stricture) tends to worsen steadily. Odynophagia (pain on swallowing) may suggest mucosal involvement (eg inflammation

Key points

Dysphagia requires initial exclusion of mucosal and structural pathology through endoscopy and imaging.

Eosinophilic oesophagitis needs to be excluded through endoscopy and biopsy, even when the oesophagus appears normal; treatment of EoE should be undertaken in a specialist clinic.

Functional oesophageal disorders should be assessed with HRM. Where OG] obstruction is suspected, further assessment with timed barium swallow should be considered.

Achalasia subtype is determined through HRM Management should be undertaken in a specialist unit which has a high turnover of treating this condition.

Treatment for achalasia includes botulinum toxin (in patients who are elderly or with morbidity burden) or with either graded pneumatic dilatation, POEM or Heller myotomy depending on patient choice and local expertise.

Management of motility disorders of the oesophageal body depends upon whether the manometric diagnosis is that of a major or minor motility disorder. A joint decision with the therapist should include conservative, endoscopic and neuromodulating therapy as well as consideration for behavioural and psychological therapy which can occur concomitantly.

KEYWORDS: dysphagia, achalasia, oesophagus, eosinophilic, manometry

DOI: 10.7861/clinmed.2021-0069 
or malignancy). ${ }^{3}$ Sudden food impaction represents a medical emergency that necessitates urgent endoscopic evaluation and therapy. Where symptoms are also associated with concerning features (such as weight loss and nutritional compromise), assessment should be prioritised (Table 1).

\section{Mechanical causes}

Malignant disorders, submucosal lesions or degenerative spinal disease can cause intraluminal or extraluminal obstruction and dysphagia. Erosive reflux disease can progress to peptic strictures and eosinophilic oesophagitis (EoE) can be associated with fibrotic narrowing of the oesophagus. Idiopathic fibrosis (Schatzki ring), complications of hiatus hernia or complications of surgery (tight/ slipped/twisted fundoplication and bariatric surgery) should also be considered. ${ }^{4}$

\section{Mucosal disease}

EoE is a chronic immune / antigen-mediated inflammatory condition of the oesophagus, which can lead to a sensation of food sticking or going down slowly even without stricture formation. It is histologically diagnosed when more than 15 eosinophils are identified per high-powered field. ${ }^{5}$

Following gastroscopy and $\mathrm{pH}$ monitoring studies, gastrooesophageal reflux disease (GORD) can be subclassified into those with mucosal pathology (erosive oesophagitis, Barrett's oesophagus or peptic stricture) or endoscopy-negative reflux disease (ENRD; normal endoscopy, pathological acid exposure and/or reflux-symptom association). Functional heartburn (FH) is diagnosed when reflux-like symptoms occur alongside normal endoscopy and $\mathrm{pH}$ monitoring. ${ }^{6}$

\section{Motility disorder}

Primary motility disorders are the consequence of abnormalities of oesophageal function. These are either due to increased contractility or spasm, reduced or absent contraction, or disorganised movement of the oesophageal body and/or the lower oesophageal sphincter. The cornerstone of assessment is high-resolution manometry (Table 2).

Achalasia is the prototypical condition of a primary motility disorder and is the consequence of a non-relaxing lower oesophageal sphincter along with absent or spastic oesophageal body function. The exact pathogenesis is poorly understood. Presenting symptoms include dysphagia to solids/liquids, chest pain, regurgitation and/or weight loss, the intensity of which are used as the basis for determining an Eckardt symptom score. ${ }^{7}$ Achalasia is a chronic, irreversible, lifelong condition that profoundly disturbs a patient's quality of life and, if left untreated, can lead to a decompensated, dilated (sometimes sigmoid) oesophagus with nutritional compromise. ${ }^{8}$

\section{Systemic diseases (rheumatologic and neurologic)}

Dysphagia can be seen in rheumatological conditions including Sjogren's syndrome, systemic lupus erythematosus, mixed connective tissue disease, rheumatoid arthritis and systemic sclerosis. It can be explained by xerostomia as well as reduction (or complete absence) of oesophageal peristalsis. Also, immunosuppressants used to treat these conditions can lead to oesophageal candidiasis. The mechanism leading to aberrant motility is unknown, though an inflammatory myopathy or neuropathy has been suggested. ${ }^{8}$

\section{Drug-induced dysphagia}

Drugs can induce dysphagia through several mechanisms: directly affecting peristalsis; xerostomia; as a consequence of pill stasis and local inflammation; and inducing GORD (Table 1). Moreover, anticholinergic and anti-dopaminergic effects of antipsychotics and tricyclic antidepressants may influence the coordination and vigour of peristalsis and lower oesophageal sphincter (LOS) function. The use of opiates in particular may intensify oesophageal contraction and/or LOS hypercontraction, sometimes even mimicking spasm and achalasia (Fig 1). ${ }^{9}$

\section{Table 1. Causes of dysphagia}

\begin{tabular}{llll} 
Luminal & Motility disorders & Systemic diseases & Drug-induced dysphagia \\
Oesophagitis & Achalasia & Rheumatoid arthritis & Antipsychotics \\
Peptic stricture & OG outflow obstruction & Scleroderma & Tricyclic antidepressants \\
Fibrous stricture & Jackhammer oesophagus & Sjogren's syndrome & Opioids \\
Schatzki ring & Distal oesophageal spasm & Systemic lupus & Iron supplements \\
Narrow calibre oesophagus & Absent peristalsis & Parkinson's disease & Potassium supplements \\
Web & Ineffective oesophageal & Cerebrovascular disease & NSAIDs \\
Diverticulum & motility & Multiple sclerosis & Tetracyclines \\
Large/distorted hiatus hernia & & Cerebellar disease & Bisphosphonates \\
Post-surgical & & Myasthenia gravis & Calcium channel blockers \\
Malignancy & & Myopathy & Nitrates \\
External compression & Motor neuron disease & Alcohol \\
Lymph nodes & & Pill stasis \\
Vertebral compression & & \\
Vascular compression & & & \\
\hline
\end{tabular}

NSAIDs = non-steroidal anti-inflammatory drugs; OGJ = oesophago-gastic junction. 
Table 2. Major motility disorders

Disorder
Achalasia
Type I (classic)
Type II (compression)
Type III (vigorous)

\section{OG] outflow obstruction}

Idiopathic

Secondary causes

\section{Major motility disorders}

Diffuse spasm

Jackhammer oesophagus

Absent peristalsis

\section{Manometric findings ${ }^{a}$}

Non-relaxing LOS + aperistalsis

Non relaxing LOS + oesophageal pressurisation

Non-relaxing LOS + increased contractile vigour

Non-relaxing LOS + normal peristalsis

Incompletely expressed achalasia, EoE, strictures, post-surgery (eg antireflux) or opioids

Relaxing LOS + premature contraction

Relaxing LOS + increased contractile vigour

Relaxing LOS + loss of peristalsis

\section{Preferred Treatment}

LHM, PD and POEM

LHM, PD and POEM

Observation, trial of botulinum toxin and PD (if diagnosis definitive)

EoE: oral steroids

Strictures: endoscopic dilation

Opioids: withdrawal or reduction

$\mathrm{CCB}$, botulinum toxin and PD

PPI, CCB/nitrates and botulinum toxin

PPI, $C C B / n i t r a t e s$ and botulinum toxin

Conservative management and PPI

${ }^{a}=$ for a detailed explanation of the parameters involved please refer to Chicago classification version $4 ;{ }^{10} \mathrm{CCB}=$ calcium channel blocker; EoE $=$ eosinophilic oesophagitis; LHM = laparoscopic hiller myotomy; LOS = lower oesophageal sphincter; OG] = oesophago-gastic junction; PD = pneumatic dilation; POEM = peroral endoscopic myotomy; PPI = proton pump inhibitors.

\section{Endoscopy}

Exclusion of a malignant, structural and mucosal disorder is a priority. In the majority of cases of EoE, classical endoscopic features include oedema, fibrotic rings (trachealisation), exudates, furrows and/or strictures. ${ }^{5,8}$ Even when appearances are apparently macroscopically normal, multiple biopsies are required from the distal, mid- and proximal oesophagus as eosinophilia can be subtle/patchy and mucosa can appear normal in up to $20 \%{ }^{5}$

\section{High-resolution manometry}

High-resolution manometry (HRM) is the gold standard modality for investigating peristalsis and LOS function. HRM produces a spatiotemporal representation of oesophageal and LOS function whereby variations in pressure are represented as colour changes over a time axis. The Chicago classification, now in its fourth iteration, is the standard to interpret and define motility disorders in a uniform and systematic way. Broadly, pathology is classified first according to oesophago-gastic junction (OGJ) morphology, then into abnormalities of the oesophageal body. ${ }^{10}$

Achalasia is the most common studied motility disorder. Alongside a non-relaxing sphincter, HRM divides achalasia into type I (absent peristalsis, commonly a later manifestation), type II (an earlier form associated with a yet non-dilated oesophagus and 'pan-oesophageal pressurisation') and type III (characterised by spastic contractions). Idiopathic OG] outflow obstruction (OGJOO) is characterised by a non-relaxing LOS but with intact oesophageal body peristalsis (Table 2). ${ }^{10}$

Where the OG] is normal, attention is shifted to oesophageal body function. Major motor disorders can induce symptoms such as dysphagia, chest pain and regurgitation. These include distal oesophageal spasm, hypercontraction and absent peristalsis. ${ }^{10}$ Minor motor disorder might be associated with similar symptoms.
Known as ineffective oesophageal motility (IEM), such disorders are due to hypotensive or frequently failed (but not absent) contraction; the wider the break in peristalsis, the more likely bolus transport is interrupted and symptoms ensue (Table 2).

The standard for HRM testing is to investigate patients by providing $5 \mathrm{~mL}$ water swallows while supine. More recently, free drinking and swallowing solids have been included, especially when subtle obstruction needs to be identified, symptoms need to be reproduced and/or to confirm normal underlying motility in those with IEM with standard testing. ${ }^{11}$

\section{Timed barium swallow, EndoFLIP and imaging}

The timed barium swallow (TBS) has become a standard test, functioning as a surrogate marker of oesophageal emptying. It measures the barium column height and width up to 5 minutes after drinking 100-200 mL of barium. The TBS can be undertaken at index and after therapy to measure success of clearance. ${ }^{12}$ Endolumenal functional lumen imaging probe (EndoFLIP; Medtronic, Dublin, Ireland) can be used to measure obstruction, as well as intraoperatively to determine the adequacy of OG] disruption. ${ }^{13}$ Comprised of impedance electrodes which run along a bag, EndoFLIP is placed across the OG] to measure cross-sectional diameter and distensibility during a sequence of distensions. Finally, endoscopic ultrasound and cross-sectional imaging might be necessary to exclude disorders associated with submucosal or external compression.

\section{Treatment}

Management of dysphagia firstly requires targeting mucosal or structural pathology. Otherwise, benign disorders commonly receive a trial of acid-reducing therapy (eg proton pump inhibitors (PPI)). EoE can be treated with twice daily PPI, swallowed topical steroids 


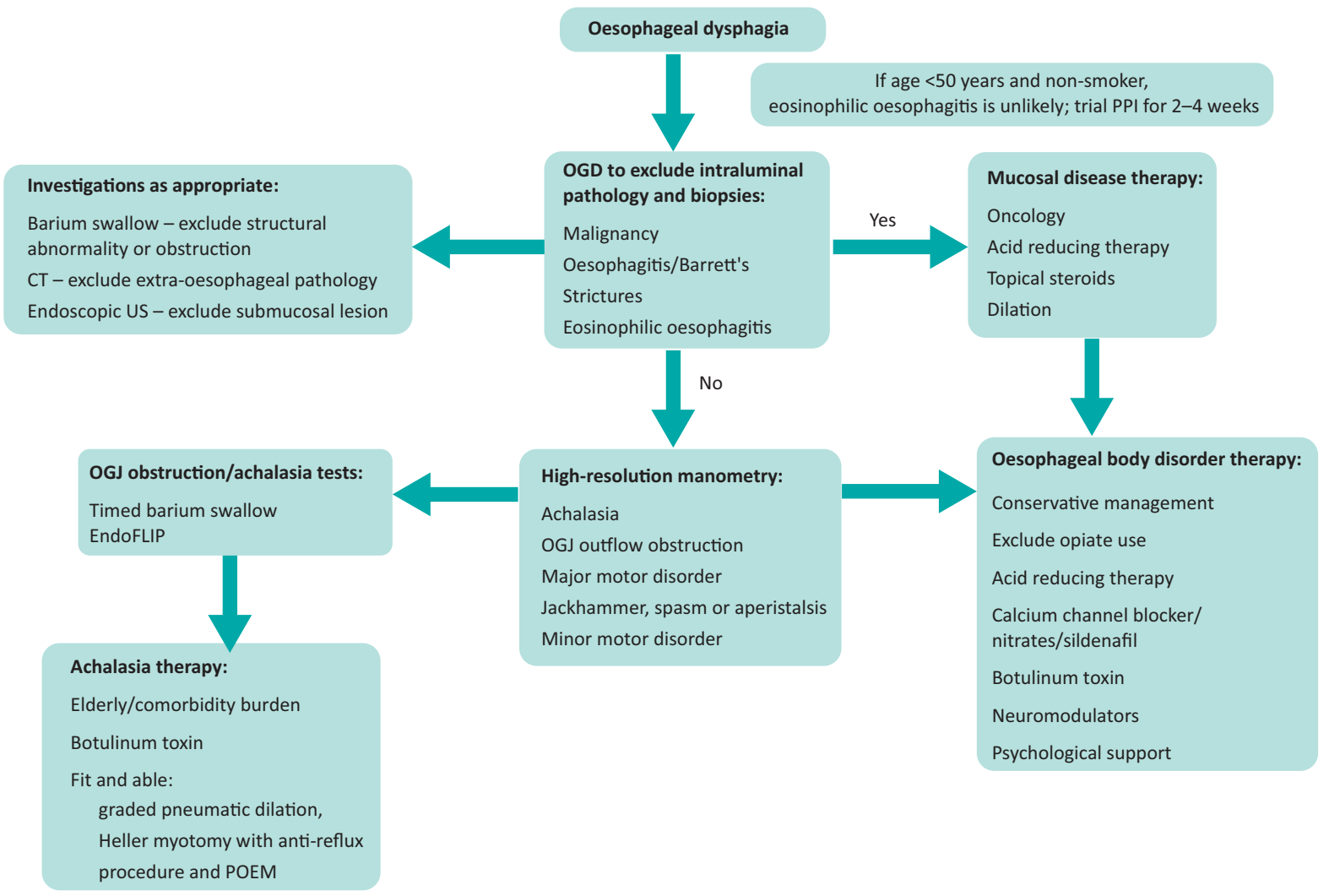

Fig 1. Diagnostic approach to dysphagia and achalasia. $C T=$ computed tomography; $O G D=$ oesophago-gastro-duodenoscopy; OG] = oesophagogastic junction; POEM = per-oral endoscopic myotomy; PPI = proton pump inhibitors; US = ultrasound.

(eg budesonide/fluticasone) or an elimination diet (under guidance of a dedicated dietitian). Endoscopic assessment is required 2-3 months after initiation of medicine or reintroduction of food groups to confirm histological response. Dilation might still be required to release associated fibrosis. Perforation rates are similar than for other disorders; one meta-analysis of 992 EOE dilations found it to be highly effective, with a perforation rate of $0.3 \%{ }^{16}$

Treatment of motility disorders is reliant upon manometry findings. Treatment for achalasia relates to comorbidity, age, achalasia subtype, patient preference and local expertise. ${ }^{14}$ Botulinum toxin injection is limited to those who cannot tolerate definitive invasive therapies; the elderly or those with comorbidities. ${ }^{14}$ Pneumatic balloon dilation has a success rate of $85 \%$ at 5 years, equivalent to Heller myotomy, but only if dilatation is performed in a graded fashion; 30 then $35 \mathrm{~mm}$ undertaken 2-3 weeks apart with the proviso that dilatation in subsequent years are permitted. ${ }^{17}$ Heller myotomy entails the surgical transection of the LOS muscle followed by a partial anti-reflux procedure. Per-oral endoscopic myotomy (POEM) also transects the LOS muscle, but is undertaken endoscopically, after creation of a tunnel through the mucosa; an anti-reflux procedure is not required. A further advantage of POEM might be in treating type III achalasia as the myotomy can reach proximal areas associated with spasm not accessible with the Heller. ${ }^{18}$ Randomised controlled trials have shown that outcomes are equivalent across all three treatment options; graded pneumatic dilatation, POEM and Heller myotomy. ${ }^{15}$
Treatment of motor disorders of the oesophageal body depends upon both manometry and symptoms. For major motor disorders associated with hypercontraction or spasm, treatment aim to reduce contraction with medicines (eg nitrates or sildenafil) or through endoscopy (eg botulinum toxin or dilatation). Absent or hypotensive motility on the other hand could incorporate conservative management (eg consuming softer food followed by fluids to help progression) or medicines (eg prokinetics). Not uncommonly, empirical acid-reducing therapy is provided concomitantly, or a formal assessment for reflux disease is considered. With all functional disorders, neuromodulators, anxiolytics and/or cognitive behavioural therapy may also be required to help treat sensitivity, manage intensity of symptoms as well address concomitant psychological sequelae. Ultimately, management of functional upper gastrointestinal disorders is rarely algorithmic, rather the best outcomes are achieved when treatment is a joint decision reached after weighing the risks and merits of every option carefully with an emphasis on patient choice.

\section{References}

1 Morris H. Dysphagia in a general practice population. Nurs Older People 2005;17:20-3.

2 Takizawa C, Gemmell E, Kenworthy ], Speyer R. A systematic review of the prevalence of oropharyngeal dysphagia in stroke, Parkinson's disease, Alzheimer's disease, head injury, and pneumonia. Dysphagia 2016;31:434-41. 
3 Cho SY, Choung RS, Saito YA et al. Prevalence and risk factors for dysphagia: a USA community study. Neurogastroenterol Motil 2015;27:212-9.

4 Eslick GD, Talley NJ. Dysphagia: epidemiology, risk factors and impact on quality of life - a population-based study. Aliment Pharmacol Ther 2008;27:971-9.

5 Mari A, Abu Baker F, Mahamid M et al. Eosinophilic esophagitis: pitfalls and controversies in diagnosis and management. Minerva Med 2020;111:9-17.

6 Gyawali CP, Kahrilas PJ, Savarino E et al. Modern diagnosis of GERD: the Lyon Consensus. Gut 2018;67:1351-62.

7 Eckardt VF, Aignherr C, Bernhard G. Predictors of outcome in patients with achalasia treated by pneumatic dilation. Gastroenterology 1992:103:1732-8.

8 Philpott H, Garg M, Tomic D, Balasubramanian S, Sweis R. Dysphagia: Thinking outside the box. World J Gastroenterol 2017;23:6942-51.

9 Stoschus B, Allescher HD. Drug-induced dysphagia. Dysphagia 1993:8:154-9.

10 Yadlapati R, Kahrilas PJ, Fox MR et al. Esophageal motility disorders on high-resolution manometry: Chicago classification version 4.0. Neurogastroenterol Motil 2021;33:e14058.

11 Sanagapalli S, McGuire J, Leong RW et al. The clinical relevance of manometric esophagogastric junction outflow obstruction can be determined using rapid drink challenge and solid swallows. Am J Gastroenterol 2021;116:280-8.

12 Rohof WO, Lei A, Boeckxstaens GE et al. Esophageal stasis on a timed barium esophagogram predicts recurrent symptoms in patients with long-standing achalasia. Am J Gastroenterol 2013;108:49-55.

13 Carlson DA, Kahrilas PJ, Lin Z et al. Evaluation of esophageal motility utilizing the functional lumen imaging probe. Am J Gastroenterol 2016;111:1726-35.

14 Pandolfino JE, Gawron AJ. Achalasia: a systematic review. JAMA 2015;313:1841-52.

15 Weusten B, Barret M, Bredenoord AJ et al. Endoscopic management of gastrointestinal motility disorders - part 1: European Society of Gastrointestinal Endoscopy (ESGE) Guideline. Endoscopy 2020;52:498-515.

16 Moawad FJ, Cheatham JG, DeZee KJ. Meta-analysis: the safety and efficacy of dilation in eosinophilic oesophagitis. Aliment Pharmacol Ther 2013;38:713-20.

17 Boeckxstaens GE, Annese V, des Varannes SB et al. Pneumatic dilation versus laparoscopic Heller's myotomy for idiopathic achalasia. N Engl J Med 2011;364:1807-16.

18 Kumbhari V, Tieu AH, Onimaru M et al. Peroral endoscopic myotomy (POEM) vs laparoscopic Heller myotomy (LHM) for the treatment of Type III achalasia in 75 patients: a multicenter comparative study. Endosc Int Open 2015;3:E195-201.

Address for correspondence: Dr Rami Sweis, University College Hospital, 235 Euston Road, London NW1 2BU, UK. Email:dr.ramisweis@gmail.com 